\title{
AN ENVIRONMENT-VISUALIZATION SYSTEM WITH IMAGE-BASED RETRIEVAL AND Distance CALCUlation METHOD
}

\author{
Yuka Toyoshima $^{1}$, Yasuhiro Hayashi ${ }^{2}$ and Yasushi Kiyoki ${ }^{1}$ \\ ${ }^{1}$ Graduate School of Media and Governance, Keio University, Kanagawa, \\ Japan \\ ${ }^{2}$ Faculity of Economics, Musashino University, Tokyo, Japan
}

\begin{abstract}
Many environmental issues have occured in this world and these issues are common to all human beings. It is considered that environmental issues caused by humans exist in the "border" between nature and human society. In other words, there is the possibility that finding the "border" leads to determine the cause of environmental issues and discover the solution. This paper presents an environment-visualization system with image-based retrieval and distance calculation method as the first step of research for finding the "border". We focused on the plastic garbage issue which is related to SDGs 14 and this study was made to find the "border", source of the plastic garbage which is scattered on the coast area. In addition, we aim to realize the system which enables people to share the knowledge about the plastic issue in order to acquire knowledge of the environment issues and to promote concrete action to realize sustainable nature and society. In this system, there are 3 features: (1) Composition-Based Image Retrieval Function, (2) SpatioTemporal-Based Mapping Function, and (3) Coast-area Location-Checking for Selected Images Function. (1) is the image retrieval function for detecting highly-related images to a query-image with dividing one image to three images for separating with nature, human society, and "border". We used euclid calculation to calculate the similarity and show the results in the ranking format. (2) is the mapping function with using the spatio-temporal information which is accompanied with images. (3) is the location-checking function to judge whether the photographing spot is near the ocean or not with image processing metric and select the images only which are near the ocean. We present several experimental results to clarify the feasibility and effectiveness of our method.
\end{abstract}

\section{KEYWORDS}

Environment-Visualization, Image-based Retrieval, Image Processing, Distance Calculation, SDGs (Sustainable Development Goals)

\section{INTRODUCTION}

Many environmental issues have occured in this world and these issues are common to all human beings. It is considered that environmental issues caused by humans exist in the "border" between nature and human society. In other words, there is the possibility that finding the "border" leads to determine the cause of environmental issues and discover the solution.

Dhinaharan Nagamalai et al. (Eds) : ACSIT, SIPM, ICITE, ITCA - 2019

pp. 131-146, 2019. C CS \& IT-CSCP 2019

DOI: $10.5121 /$ csit.2019.90312 
In this paper, we present an environment-visualization system with image-based retrieval and distance calculation method as the first step of research for finding the "border". This method retrieve the images which is divided with 3 parts: nature, human society, and the border between nature and human society. This system enables to recognize the "meaning" of the envrionmental situations and phenomena from each images and searches the images which is similar with a query-image as a human with adopting the method of division of images with 3 parts based on composition. Therefore, this system enables to detect the specific environmental situation and phenomenon corresponding to the user's imagination. In addition, this method calculates the distance between the photographing spot and object with metadata accompanied with images and reflects the calculation results to the result of image retrieval. Therefore, it is possible to search more similar images than previous image retrieval.

In this paper, we focused on the issue of plastic garbage as the environmental issue to work on. Plastic garbage issue is getting worse year by year. According to the report about marine debris which was announced in World Economic Forum Annual meeting in Davos [1], at least 8 million ton of plastics end up in the ocean. This is equal to one garbage truck of plastics ends up in the ocean every minute. If we do not do anything, or do not try to solve this problem, the amount of plastics which flow into the ocean will be 2times in 2030 and 4 times in 2050. Figure 1 shows the yearly comparison of the amount of plastics flowing into the ocean in one year.

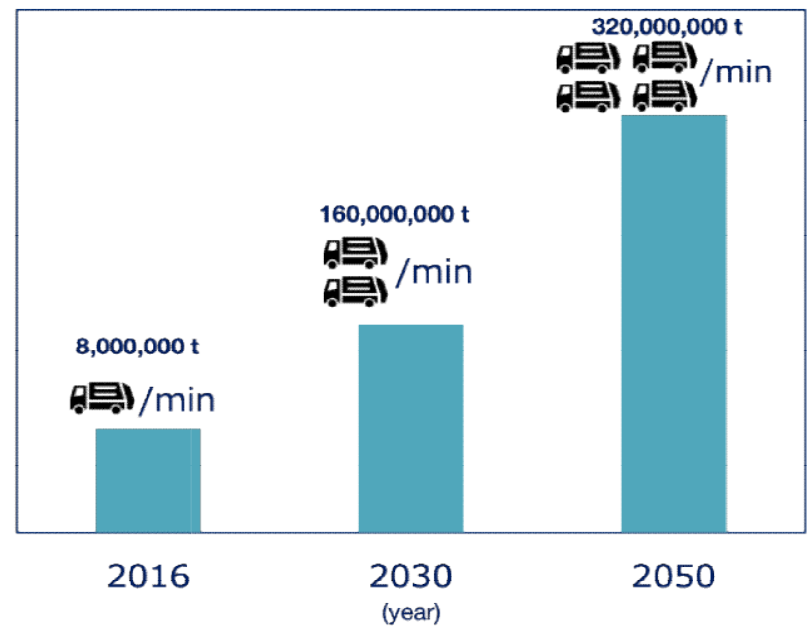

Figure 1. The amount of plastics which flow into the ocean in one year [1]

For this reason, in this paper, we set the issue as "how to reduce marine garbage and microplastic". This issue is corresponding to SDGs 14. Sustainable Development Goals (SDGs) [2] are summarized about environment issues. Future Learning should support SDGs in order to acquire knowledge of the environment issues and to promote concrete action to realize sustainable nature and society. SDGs are 17 goals defined by United Nations to solve the issues about society and environment until 2030. Figure 2 shows the goals of SDGs.

1. No Poverty

2. Zero Hunger

3. Good Health and Well-Being

4. Quality Education

5. Gender Equality 
6. Clean Water and Sanitation

7. Affordable and Clean Energy

8. Decent Work and Economic Growth

9. Industry, Innovation and Infrastructure

10. Reduced Inequalities

11. Sustainable Cities and Communities

12. Responsible Composition and Production

13. Climate Action

14. Life Below Water

15. Life On Land

16. Peace, Justice and Strong Institutions

17. Partnerships for the Goals

Figure 2. SDGs: Sustainable Development Goals [2]

In this paper, we propose the method leading to the solution of the problem by the method of image processing. In addition, we aim to realize the system which enables people and organization to share the seriousness and knowledge of plastic garbage issue and to discuss and take some action for solving or improving the issue.

\section{RELATED WORK}

In this section, we would introduce the researches related our study.

\subsection{D WORLD MAP SYSTEM}

There is the system of image retriever and visualization about environment: " $5 \mathrm{D}$ World Map" [3][4][5][6][7]. 5D World Map system [3][4][5][6][7] is a collaborative knowledge sharing system that enables to create and share knowledge by visualizing with a map. This system analyses multimedia such as images, videos, audio, documents, etc., by semantic, temporal and spatial information. In addition, this system integrates and visualizes the analyzed results as a 5- dimentional dynamic historic atlas (5D World Map Set). The main feature of this system is to create various context-dependent patterns of environmental/historical/cultural stories according to a user's viewpoints dynamically. For example, when users would like to find the images about forest fire and understand the place and time of the phenomenon of forest fire, they can search by keywords, images, or setting the target databases.

The composition of images is promising to be supported in 5D World Map, as the image retriever of this system. This system enables to realize that users find out the images that show the particular situation of environment such as "forest fire happened in the night".

\subsection{IMAGE QUERY CREATION Method}

There is an image query creation method of using multiple images [8][9]. This method creates the query image to express user's intentions by combining the multiple images based on colors and shapes of objects. This method enables users to express their imagination and intention by multiple images and create the exact image query for each user.

Our method focuses on the particular environmental situation and phenomenon as user's imagination and intention. 


\section{The Concept And Structure Of OUR System}

In this section, we explain about the concept and structure of our system. Figure 3 shows the concept of our system.

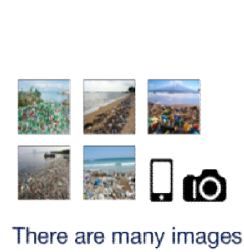

with plastic garbage

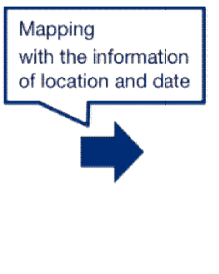

Judge whether the photographing spot
is near the sea or not

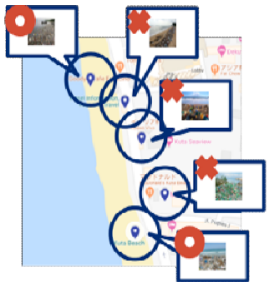

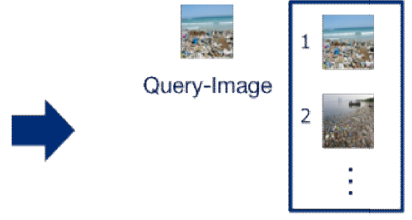

Just select the images which is near the ocean and reflect in the result of a composition-based image retrieval

Figure 3. The concept of our system

There are many images with plastic garbage and also these images have the information of plastic such as the information of location and date. With these information, this system mapping the images like this and judging whether the distance between the photographing spot and the sea is close or not. The blue pin is the photographing spot and it is the center of this circle with a radius $r$. If the color of ocean is included in the circle, this system judge "This spot is near the sea". If not, "This spot is far from the sea".

This system just selects the images which is near the ocean and reflects in the result of a composition-based image retrieval.

In addition, Figure 4 shows the system structure.

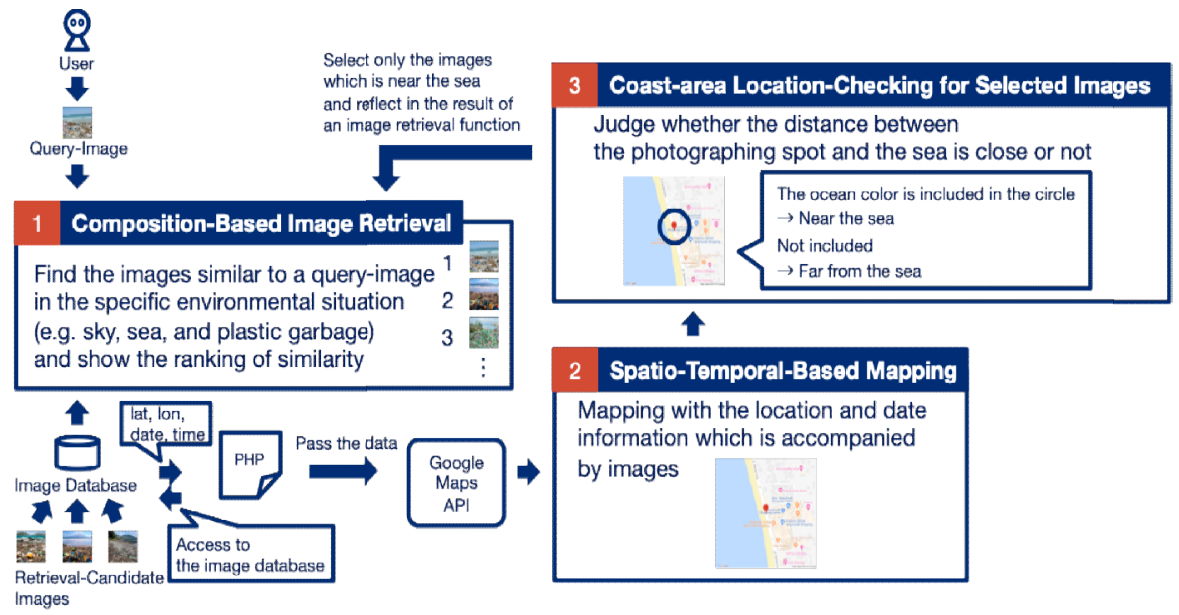

Figure 4. System Structure

In this system, there are 3 features: (1) Composition-Based Image Retrieval Function, (2) Spatio-Temporal-Based Mapping Function, and (3) Coast-area Location-Checking for Selected Images Function.

(1) is the image retrieval function for detecting highly-related images to a query-image with dividing one image to three images for separating with nature, human society, and "border". We used euclid calculation to calculate the similarity and show the results in the ranking 
format. (2) is the mapping function with using the spatio-temporal information which is accompanied with images. (3) is the location-checking function to judge whether the photographing spot is near the ocean or not with image processing metric and select the images only which are near the ocean.

\section{An EnVironment-Visualizatoin System With Image-BaSed Retrieval ANd Distance Calculation Method}

In this section, we would mention the implement of our method.

In this method, each "retrieval-candidate image" is divided into three parts, Top-Part, Middle- Part and Bottom-Part. A "query-image" is also constructed with three parts as TopPart, Middle- Part and Bottom-Part. When the image retrieval is applied, this method compares each corresponding part between query-image and retrieval-candidate image by correlation computing, and makes ranking of retrieval-candidate images according to correlations. In addition, our method creates the map image in which Google Maps [10] makes a pin with the spatio-temporal information of the image and to judge whether the photographing spot is near the ocean or not with image processing metric.

The steps of detailing our method are as follows:

\section{1) Decide retrieval-candidate images $(\mathrm{m})$}

We decided retrieval-candidate images $(\mathrm{m})$ as the retrieval objects.

2) Decide the number (n) of colors which we handle and make a cluster of $\mathbf{n}$ colors

We defined the number ( $\mathrm{n}$ ) of colors and created the cluster of $\mathrm{n}$ colors to standardize colors of $\mathrm{m}$ images.

3) Divide one image into three images

We divided one image $(300 \times 300$ pixels $)$ into three images to consider the composition of each image. Figure 5 shows the example of divided-part-images in a single image.

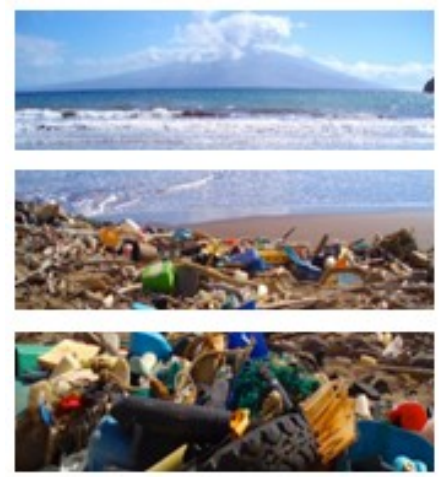

Figure 5. Divided-part-images in a single image

In this method, each image is divided into Top-Part, Middle-Part, and Bottom-Part.

4) Create a color histogram with each divided-part-image

We created a color histogram with each divided-part-image. We extracted each pixel color from each image and put it in RGB space. Then, we converted RGB space to HSVspace. Equation 
(1) (5) show the formula to convert RGB into HSV. The range of RGB values is 0 to 1 . In addition, max and min are the maximum and minimum values of RGB

$$
\begin{aligned}
& H=\frac{G-B}{\max -\min } \times 60, I F \max =R \\
& H=\frac{B-R}{\max -\min } \times 60+120, I F \max =G \\
& H=\frac{R-G}{\max -\min } \times 60+240, I F \max =B \\
& S=\frac{\max -\min }{\max } \\
& V=\max
\end{aligned}
$$

After that, for the distance calculation in HSV space, we used the Godlove color difference formula [11]. Equation (6) shows Godlove color difference formula.

$$
\Delta \text { godlove }\left(H_{1}, S_{1}, V_{1}, H_{2}, S_{2}, V_{2}\right)=\frac{2 S_{1} S_{2}\left(1-\cos \left(2 \pi \frac{\left|H_{1}-H_{2}\right|}{100}\right)\right)+\left(\left|S_{1}-S_{2}\right|\right)^{2}+\left(4\left|V_{1}-V_{2}\right|\right)^{2}}{2}
$$

\section{5) Use Euclid Distance Calculation}

We used the Euclid distance calculation to show the color distance between a query-image and retrieval-candidate images (m). Equation (7) is the Euclid distance calculation.

$$
h 1, h 2, h 3=\sum_{i=1}^{n} \sqrt{\left(C_{q i}-C_{m i}\right)^{2}}
$$

Each value of color histograms for divided-part-images $(h 1, h 2, h 3)$ is calculated by the sum of the color distance between a query-image $\left(c_{q i}\right)$ and retrieval-candidate images $(m)\left(c_{m i}\right)$. The smaller result is the higher similarity.

6)Use the method to integrate each value of color histogram for divided-part-images

We used the method to integrate each value of color histogram for divided-part-images. Equation (8) is a calculation to integrate them.

$$
T h=h 1+h 2+h 3
$$

Total value of color histograms $(T h)$ is calculated by the sum of each value of color histograms for divided-part-images $(h 1, h 2, h 3)$.

\section{7) Use the method of weighting}

We used the method of weighting to emphasize the feature part (plastic garbage part) in images. Equation (9) is the calculation to multiple the weight of each part.

$$
\text { Th with weight }=h 1 \times w 1+h 2 \times w 2+h 3 \times w 3
$$

Each value of color histogram for divided images (h1, h2, h3) is multiplied by each weight $(\mathrm{w} 1, \mathrm{w} 2, \mathrm{w} 3)$. 
8) Mapping images onto a map and capturing the screen image

The retrieved images that are included latitude and longitude information as image metadata are mapped onto a map. In this system, Google Maps is applied for mapping. Afterwards, this system captures a screen image of Google Maps which makes a pin in a map.

\section{9) Judging a coastal area with location information by image-processing}

We extracted the color of each pixel from the map image created in the step 8) and judged the image was taken in the coastal area which is near the sea or not. As a judging method, this system judge based on whether the color of the ocean is included in the circle whose radius is $r$ and center point is the photographing spot.

\section{EXPERIMENTS AND DISCUSSION}

In this section, we would mention experiments and discussion.

We conducted three experiments. Experiment 1 has two experiments. Experiment 1-1 is the experiment to compare the previous system without composition and our system with composition. Experiment 1-2 is the experiment to verify the weighting. Experiment 2 is the basic experiment for judgment of image composition automatically. Experiment 3 is the basic experiment for verifying whether the distance sense of the human being when they see the map image and the system judgment are close or not.

\subsection{Experiment 1}

In this experiment, we constructed an own image database and retrieve images by following the steps as mentioned above. We decided 50 retrieval-candidate images. Figure 6 shows all retrieval-candidate images.

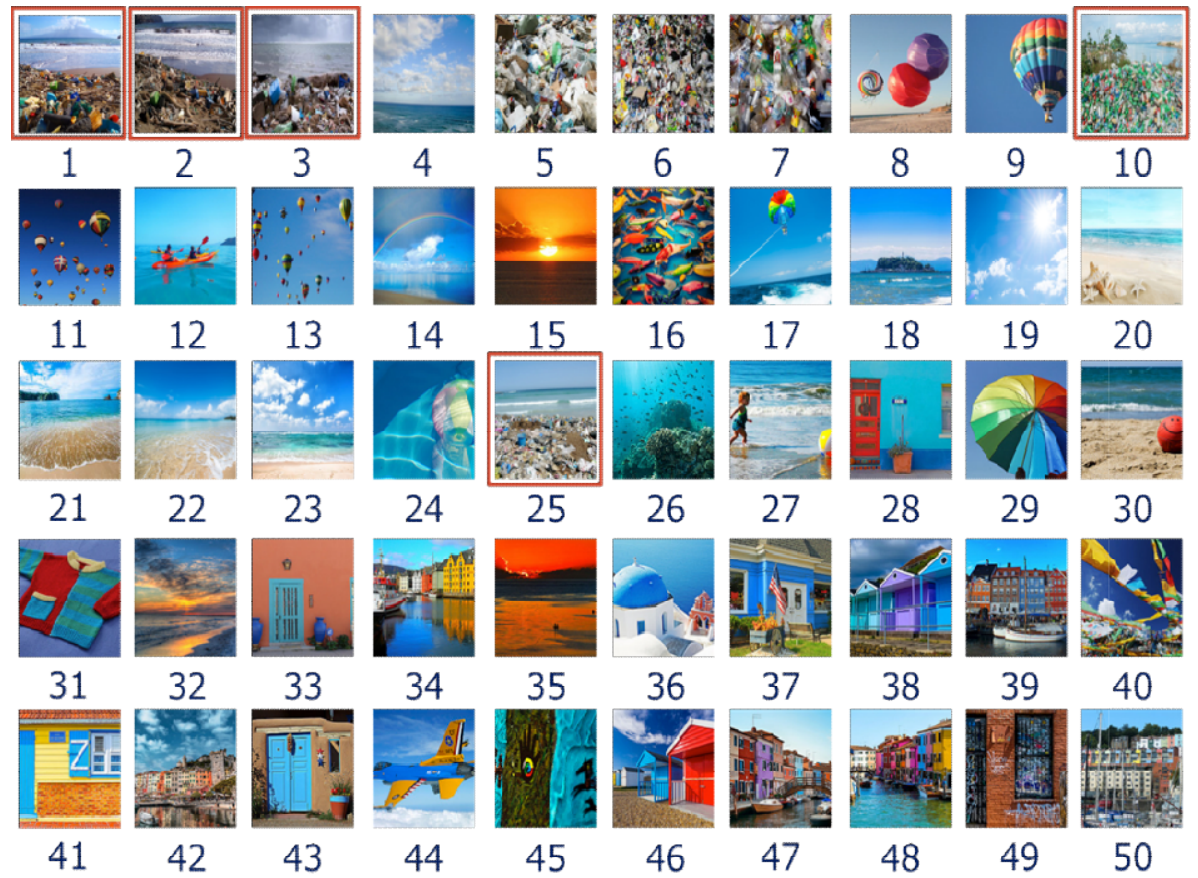

Figure 6. Retrieval-candidate images in Experiment 1 
We selected these images by Flickr [12] and Google Images [13]. We treated 5 images within 50 retrieval-candidate images as expected images to be retrieve in this experiment. We defined expected images as the images which are composed with sky, sea, and plastic garbage scattered on the beach. From figure 6 , the image surrounded by red rectangle is expected image.

We selected other images according to the following conditions:

- The image with only plastic garbage

- The image excluded plastic garbage

- The image excluded plastic garbage with different color of sky

- The image which has multiple colors in top or center region

Additionally, we handled 130 colors based on Color Image Scale [14].

There are two parts in this experiment.

\subsubsection{Experiment 1-1: Comparing With Composition and Without Composition}

In this experiment, we compare the previous system without composition and our system with composition.

Table 1 and 2 show the results of the top-ten ranking of 50 retrieval-candidate images. We used the id number 25 as a query-image and make the best similarity on purpose. In addition, we did not use the weighting method in this experiment.

Table 1 shows the result of divided-part-images.

Table 1. Result of divided-part-images

\begin{tabular}{|r|r|}
\hline img_id & \multicolumn{1}{|c|}{ Th } \\
\hline 25 & 0 \\
\hline 10 & 0.715803303 \\
\hline 1 & 0.833790638 \\
\hline 6 & 0.841656171 \\
\hline 5 & 0.86161129 \\
\hline 7 & 0.895032013 \\
\hline 3 & 0.989218226 \\
\hline 37 & 0.991339542 \\
\hline 50 & 0.991339542 \\
\hline 42 & 1.024072877 \\
\hline
\end{tabular}

There are 2 columns, img_id and Th. Img_id is the id number of each image, and Th is the sum of color histogram values of divided-part-images. Blue color is the id number of expected images. From Table 1, we can understand that the most similar image is 25 . There are also 10 in the second, 1 in the third, and 3 in the seventh.

Table 2 shows the result of non-divided images. 
Table 2. Result of non-divided-images

\begin{tabular}{|r|r|}
\hline img_id & \multicolumn{1}{|c|}{$\boldsymbol{T h}$} \\
\hline 25 & 0 \\
\hline 10 & 0.156998147 \\
\hline 1 & 0.163591752 \\
\hline 4 & 0.208711166 \\
\hline 37 & 0.218768187 \\
\hline 6 & 0.220653618 \\
\hline 7 & 0.223305956 \\
\hline 3 & 0.225188325 \\
\hline 5 & 0.227740912 \\
\hline 27 & 0.24074918 \\
\hline
\end{tabular}

There are 2 columns, img_id and H. Img_id is the id number of each image, and H is the sum of color histogram values of a non-divided image. Blue color is the id number of expected images. From Table 2 we can understand that the most similar image is 25 . There are also 10 in the second, 1 in the third, and 3 in the eighth.

Comparing with Table 1 and Table 2, it is able to say that the images which has similar color variance value be reflected to the result because it is possible to detect the volume of the color variance value for each part to divide with 3 parts based on the image composition.

\subsubsection{Experiment 1-2: Verifing Weighting}

In this experiment, we verify the method of weighting to show how the ranking is going to be changed.

We changed the ratio of weight for each part. The ratio of weight for each part is $1: 1: \mathrm{N}$ and $1: 2: \mathrm{N}$ $(\mathrm{N}>2)$ to emphasize the bottom part (the plastic garbage part).

We have 11 ways to weighting. However, we show the 4 results as Table $3 \sim 6$ in this paper. Table $3 \sim 6$ show the results of the top-ten ranking of 50 retrieval-candidate images. We used the id number 25 as a query-image and make the best similarity on purpose.

Table 3 shows the result of the case of 1:1:2.

Table 3. Result of the case of $1: 1: 2$

\begin{tabular}{|r|r|}
\hline img_id & \multicolumn{1}{|c|}{$\boldsymbol{T h}$} \\
\hline 25 & 0 \\
\hline 10 & 0.20612656 \\
\hline 6 & 0.234365182 \\
\hline 5 & 0.246331578 \\
\hline 7 & 0.261774284 \\
\hline 1 & 0.263186217 \\
\hline 37 & 0.292710457 \\
\hline 42 & 0.297620847 \\
\hline 3 & 0.299138796 \\
\hline 50 & 0.30366452 \\
\hline
\end{tabular}


There are 2 columns, img_id and Th with weight. Img_id is the id number of each image, and Th with weight is the sum of color histogram values of divided-part-images with weight. Blue color is the id number of expected images. From Table 3, we can understand that the most similar image is 25 . There are also 10 in the second, 1 in the third, and 3 in the nineth.

Table 4 shows the result of the case of 1:1:6.

Table 4. Result of the case of 1:1:6

\begin{tabular}{|r|r|}
\hline img_id & \multicolumn{1}{|c|}{$\boldsymbol{T h}$} \\
\hline 25 & 0 \\
\hline 10 & 0.15741475 \\
\hline 6 & 0.16508487 \\
\hline 5 & 0.1850233 \\
\hline 7 & 0.206919704 \\
\hline 40 & 0.228159007 \\
\hline 42 & 0.232015678 \\
\hline 37 & 0.236106372 \\
\hline 1 & 0.241070224 \\
\hline 50 & 0.247229191 \\
\hline
\end{tabular}

There are 2 columns, img id and Th with weight. Img id is the id number of each image, and Th with weight is the sum of color histogram values of divided-part-images with weight. Blue color is the id number of expected images. From Table 4, we can understand that the most similar image is 25 . There are also 10 in the second and 1 in the nineth.

Table 5 shows the result of the case of 1:2:3.

Table 5. Result of the case of 1:2:3

\begin{tabular}{|r|r|}
\hline img_id & \multicolumn{1}{|c|}{$\boldsymbol{T h}$} \\
\hline 25 & 0 \\
\hline 10 & 0.289174828 \\
\hline 6 & 0.293847877 \\
\hline 5 & 0.333508466 \\
\hline 7 & 0.38134804 \\
\hline 40 & 0.428124782 \\
\hline 42 & 0.428125157 \\
\hline 37 & 0.441694257 \\
\hline 50 & 0.456333111 \\
\hline 47 & 0.462797156 \\
\hline
\end{tabular}

There are 2 columns, img_id and Th with weight. Img_id is the id number of each image, and Th with weight is the sum of color histogram values of divided-part-images with weight. Blue color is the id number of expected images. From Table 5, we can understand that the most similar image is 25 . There are also 10 in the second. 
Table 6 shows the result of the case of 1:2:7.

Table 6. Result of the case of 1:2:7

\begin{tabular}{|r|c|}
\hline img_id & \multicolumn{1}{c|}{$\boldsymbol{h}$} \\
\hline 25 & 0 \\
\hline 10 & 0.151764309 \\
\hline 6 & 0.157147815 \\
\hline 5 & 0.175362075 \\
\hline 7 & 0.198395799 \\
\hline 42 & 0.223592992 \\
\hline 40 & 0.226733782 \\
\hline 37 & 0.229116097 \\
\hline 1 & 0.235441637 \\
\hline 50 & 0.235641094 \\
\hline
\end{tabular}

There are 2 columns, img_id and Th with weight. Img_id is the id number of each image, and Th with weight is the sum of color histogram values of divided-part-images with weight. Blue color is the id number of expected images. From Table 6, we can understand that the most similar image is 25 . There are also 10 in the second and 1 in the nineth.

From the experimental results, it is able to say that the part to be emphasized is emphasized properly by weighting in our system. In addition, we found the maximum value of weighting. In the case of $1: 1: \mathrm{N}, \mathrm{N}=6$ is the maximum value, and in the case of $1: 2: \mathrm{N}, \mathrm{N}=7$ is the maximum value.

\subsection{Experiment 2}

This experiment is the basic experiment for judgment of image composition automatically. In this experiment, we constructed an own image database and retrieve images by following the steps as mentioned above. We decided 75 retrieval-candidate images which are adding 25 images to the retrieval-candidate images of Experiment 1. Figure 7 shows all retrieval-candidate images.

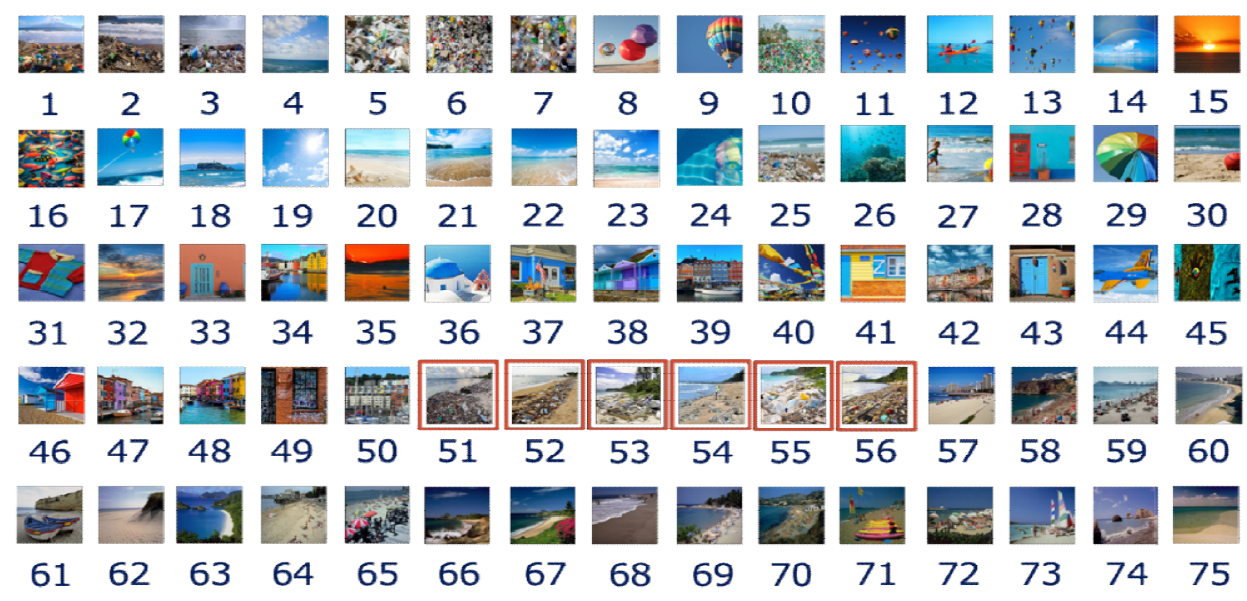

Figure 7. Retrieval-candidate images in Experiment 2 
We selected these images by Google Images [13] and test images [15] which are usually used for the image processing research. We treated 6 images within 75 retrieval-candidate images as expected images to be retrieve in this experiment. We defined expected images as the images which are composed with sky, sea, and plastic garbage scattered on the beach and taken from left diagonal. From figure 7, the image surrounded by red rectangle is expected image. We selected other images according to the conditions which excluded plastic garbage.

In addition, we defined the 16 composition patterns. Figure 8 shows the all patterns of image composition.
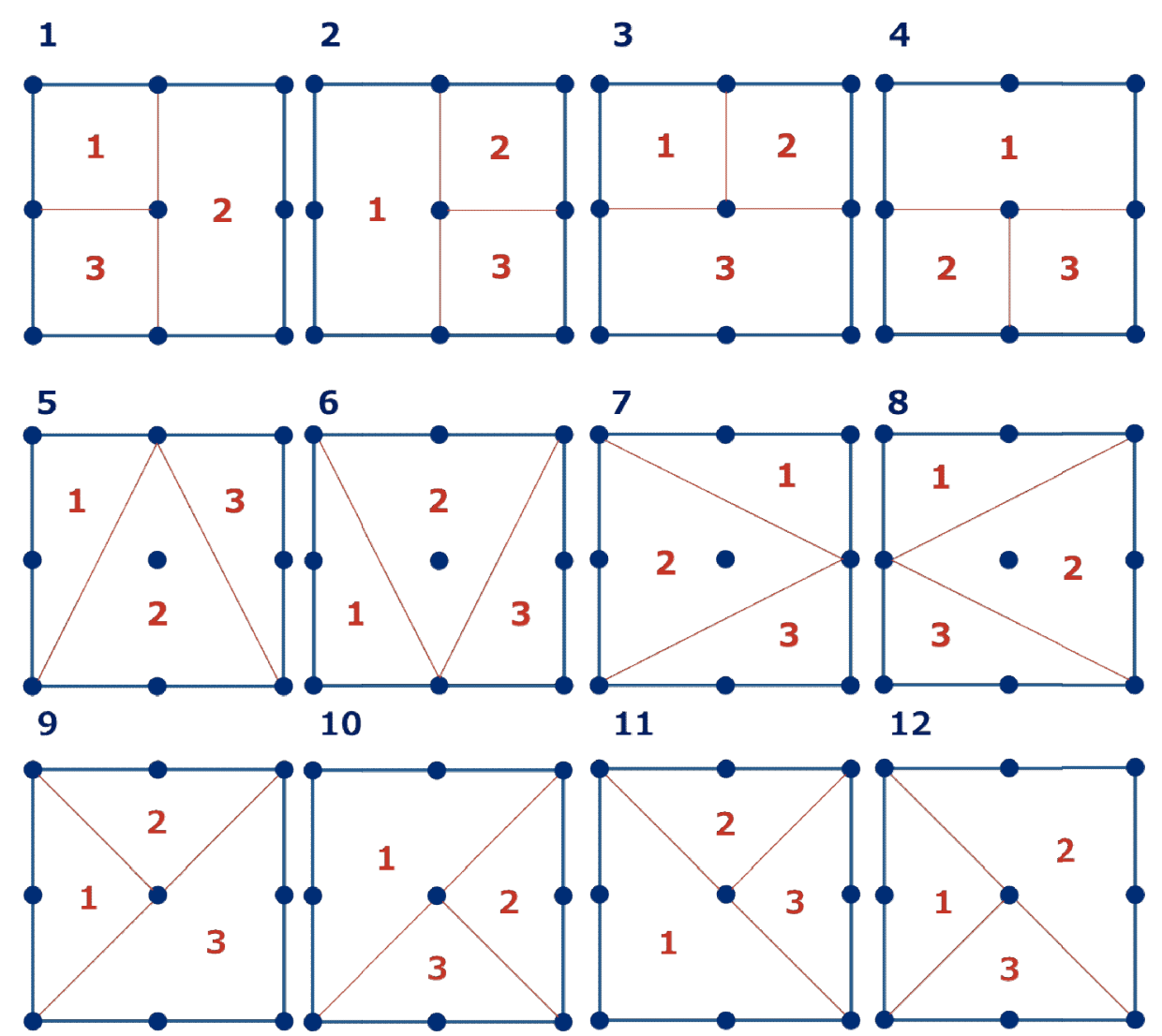

13

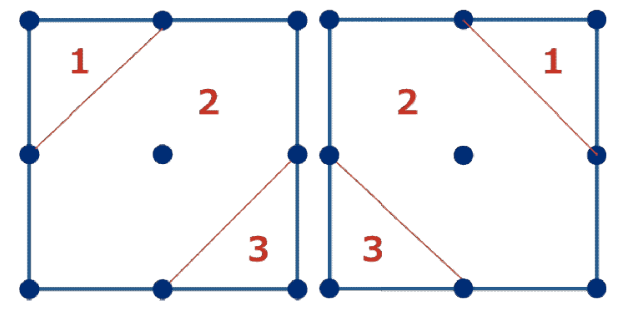

15

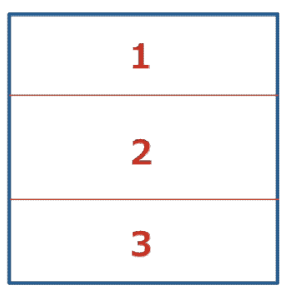

16

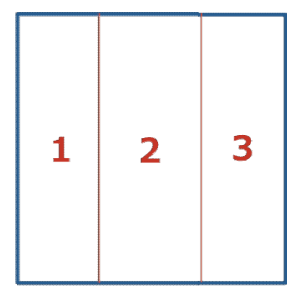

Figure 8.16 composition patterns

Top part is 1 , middle part is 2 , and bottom part is 3 . Pattern 15 and 16 are the exception case. However, we included the composition patterns because they are basic one as image composition. 
We have 16 results. However, we show the 3 results which have higher precision as Table 7 9 in this paper. Table 7 9 show the results of the top-ten ranking of 75 retrieval-candidate images. We used the id number 51 as a query-image and make the best similarity on purpose. In addition, we define composition pattern 7 is the best way to divide and search the expected images.

Table 7 shows the results of composition pattern 7 .

Table 7. Result of composition pattern 7

\begin{tabular}{|r|r|}
\hline img_id & \multicolumn{1}{|c|}{$\boldsymbol{T h}$} \\
\hline 51 & 0 \\
\hline 7 & 0.54080188 \\
\hline 5 & 0.618459439 \\
\hline 6 & 0.620064148 \\
\hline 2 & 0.641678675 \\
\hline 3 & 0.643087056 \\
\hline 50 & 0.696883611 \\
\hline 65 & 0.737541973 \\
\hline 56 & 0.761567507 \\
\hline 37 & 0.772267028 \\
\hline
\end{tabular}

There are 2 columns, img_id and Th. Img_id is the id number of each image, and Th is the sum of color histogram values of divided-part-images. Blue color is the id number of expected images. From Table 7, we can understand that the most similar image is 51. There are also 56 in the nineth.

Table 8 shows the results of composition pattern 13 .

Table 8. Result of composition pattern 13

\begin{tabular}{|r|r|}
\hline img_id & \multicolumn{1}{|c|}{$\boldsymbol{T h}$} \\
\hline 51 & 0 \\
\hline 6 & 0.597303954 \\
\hline 7 & 0.700849855 \\
\hline 2 & 0.710111445 \\
\hline 3 & 0.784299714 \\
\hline 5 & 0.81356392 \\
\hline 50 & 0.821740533 \\
\hline 37 & 0.874531216 \\
\hline 55 & 0.878133988 \\
\hline 42 & 0.882020918 \\
\hline
\end{tabular}

There are 2 columns, img id and Th. Img_id is the id number of each image, and Th is the sum of color histogram values of divided-part-images. Blue color is the id number of expected images. From Table 8, we can understand that the most similar image is 51 . There are also 55 in the nineth. 
Table 9 shows the results of composition pattern 14.

Table 9. Result of composition pattern 14

\begin{tabular}{|r|r|}
\hline img_id & \multicolumn{1}{|c|}{$\boldsymbol{T h}$} \\
\hline 51 & 0 \\
\hline 7 & 0.705883744 \\
\hline 5 & 0.726226913 \\
\hline 3 & 0.733439106 \\
\hline 70 & 0.75626755 \\
\hline 65 & 0.787828013 \\
\hline 2 & 0.815442295 \\
\hline 6 & 0.818843453 \\
\hline 56 & 0.842761986 \\
\hline 37 & 0.844634256 \\
\hline
\end{tabular}

There are 2 columns, img_id and Th. Img_id is the id number of each image, and Th is the sum of color histogram values of divided-part-images. Blue color is the id number of expected images. From Table 9, we can understand that the most similar image is 51. There are also 56 in the nineth.

Comparing the results of each composition pattern, it is able to say that our system does not detect the features of images with high precision. To improve this, it is necessary to acquire feature quantity from line component with hough transform technology.

\subsection{Experiment 3}

This experiment is the basic experiment for verifying whether the distance sense of the human being when they see the map image and the system judgment are close or not.

We defined the photographing spot as Kuta Beach in Bali island and set the longtitude and latitude as $\{-8.71669,115.168583\}$. We obtained the map image of $2560 \times 1380$ pixels whose scale is 1/100 meters. Figure 9 shows the map image we used in this experiment.

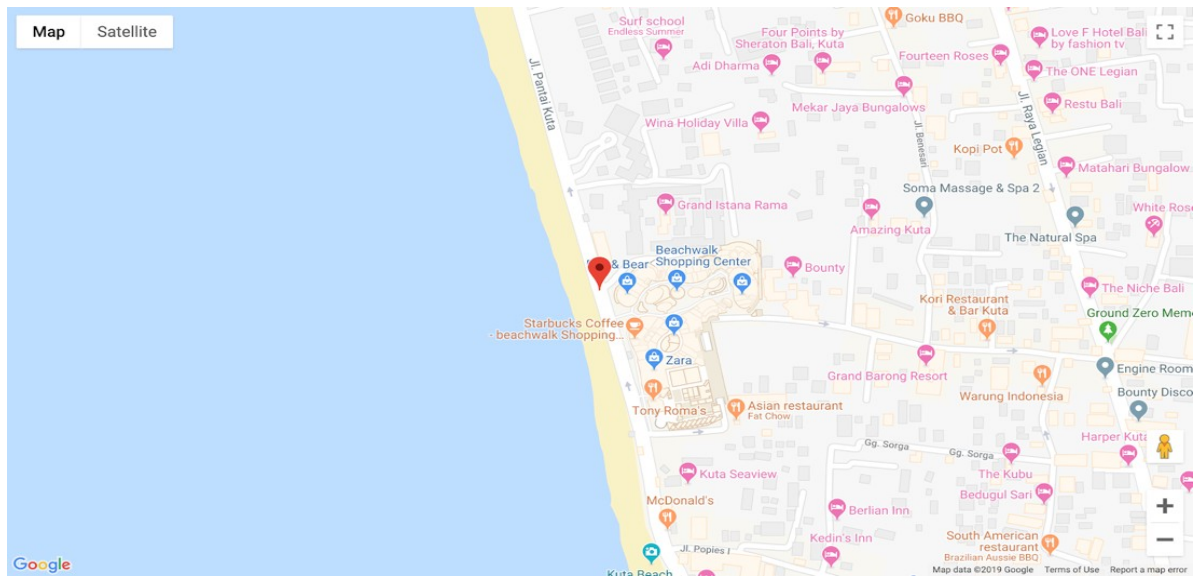

Figure 9. Map image of Kuta Beach 
In addition, we defined radius $r$ within 200 pixels. We set the color to detect based on RGB as $\mathrm{RGB}=(191,222,254)$. If this color is included in the circle, the console shows "Ocean!". If not, the console shows "Not Ocean!".

We judge the distance between photographing spot and ocean is close and we verify whether the distance sense of the human being when they see the map image and the system judgment are close or not.

The console showed "Ocean!". In other words, our system judge "the photographing spot is near the ocean". Figure 10 shows the result of console.

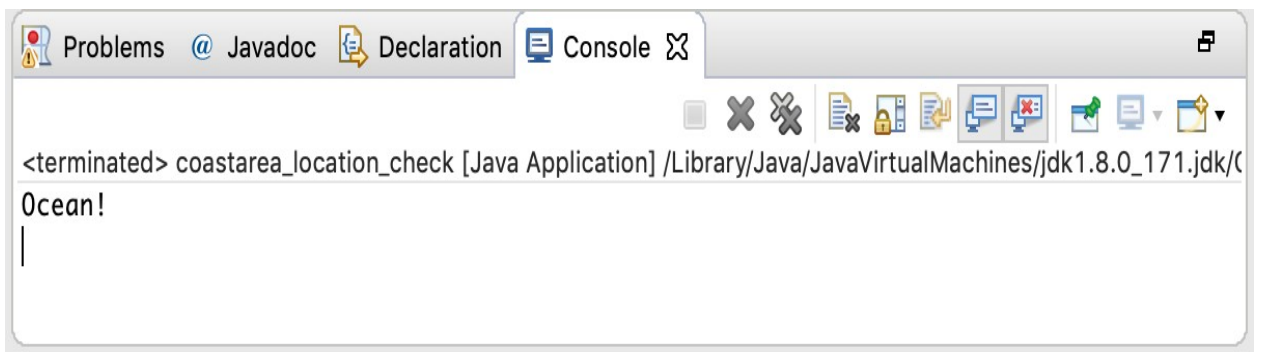

Figure 10. Result of Console

From the experiment result, it is able to say that the distance sense of the human being when they see the map image and the system judgment are close.

However, we have 2 points to improve the precision. The first point is to make it possible to deal with other scales of map images for changing the pixels of radius $r$. The second point is to acquire automatically metadata such as location information from the image database.

\section{CONCLUSiOn AND Future Works}

In this paper, we have presented an environment-visualization system with image-based retrieval and distance calculation method. We have shown about 3 functions of this system: (1) Composition-Based Image Retrieval Function, (2) Spatio-Temporal-Based Mapping Function, and (3) Coast-area Location-Checking for Selected Images Function. In addition, we have presented several experimental results about 3 functions to clarify the feasibility and effectiveness of our method.

From the experimental results and discussion, we obtained 4 opinions as follows:

- Our method using image processing metrics enables to discover the plastic garbage in coastal area

- the images which has similar color variance value be reflected to the result because it is possible to detect the volume of the color variance value for each part to divide with 3 parts based on the image composition

- the part to be emphasized is emphasized properly by weighting in our system

- there is roon for improvement regarding experiment 2 and 3 
As future works, we will make this system be able to acquire feature quantity from line component with hough transform technology. In addition, we will make this system be able to deal with other scales of map images for changing the pixels of radius $r$ and to acquire automatically metadata such as location information from the image database to improve the precision.

\section{REFERENCES}

[1] The new plastic Economy Rethinking the future of plastics, http://www3.weforum.org/docs/WEF_The_New_Plastics_Economy.pdf

[2] 17 Sustainable Development Goals (SDGs), https://www.un.org/sustainabledevelopment/sustainabledevelopment-goals/

[3] Kiyoki, Y., Kitagawa, T., and Hayama, T., "A metadatabase system for semantic image search by a mathematical model of meaning,” ACM SIGMOD Record, vol.23 no.4 pp.34-41, 1994.

[4] Kiyoki, Y., Chen, X., Sasaki, S., and Koopipat, C., "Multi-Dimensional Semantic Computing with Spatial-Temporal and Semantic Axes for Multi-spectrum Images in Environment Analysis", Information Modelling and Knowledge Bases, Vol. XXVII, IOS Press, pp.14-30, 2016.

[5] Sasaki, S., Takahashi, Y., and Kiyoki, Y., "The 4D World Map System with Semantic and Spatiotemporal Analyzers", Information Modelling and Knowledge Bases, Vol.XXI, IOS Press, pp.1 $18,2010$.

[6] Sasaki, S, and Kiyoki, Y., "Analytical Visualization Function of 5D World Map System for MultiDimensional Sensing Data", Information Modelling and Knowledge Bases, Vol. XXIX, IOS Press, pp. 71-89, 2018.

[7] Nguyen, D. T. N, Sasaki, S., and Kiyoki, Y., "5D World PicMap: Imagination-based Image Search System With Spatiotemporal Analyzer", Proceeding of The IASTED e-society 2011 Conference, Avila, Spain, pp. 271- 278, 2011.

[8] Hayashi, Y., Kiyoki, Y., and Chen, X., "An Image-Query Creation Method for Expressing User's Intentions by Combining Multiple Images", Information Modelling and Knowledge Bases, Vol.XXI, IOS Press, pp. $188-207,2010$.

[9] Hayashi, Y., Kiyoki, Y., and Chen, X., "A Combined Image-Query Creation Method for Expressing User's Intentions with Shape and Color Features in Multiple Digital Images", Information Modelling and Knowledge Bases, Vol. XXII, IOS Press, pp. 258 - 277, 2011.

[10] Google Maps, https://www.google.com/maps

[11] Godlove, I. H., "Improved Color Difference Formula, with Applications to the Perceptibility and Acceptability of Fadings", The Journal of the Optical Society of America (JOSA), Vol.41, Num.11, pp.760-772, 1951.

[12] Flickr, https://www.flickr.com/

[13] Google Image, https://images.google.com/

[14] Kobayashi, S., "Color Image Scale", The Nippon Color \& Design Research Institute ed.,Kodansha International, 2001.

[15] Content Based Image Retrieval / Image Database Search Enging, http://wang.ist.psu.edu/docs/related/ 